\title{
Glucose-dependent insulinotropic peptide and risk of cardiovascular events and mortality: a prospective study
}

\author{
Amra Jujić $^{1,2}$ (D) Naeimeh Atabaki-Pasdar ${ }^{3}$ • Peter M. Nilsson ${ }^{3}$ - Peter Almgren ${ }^{3,4}$ • Liisa Hakaste H,6,7,8 $^{\text {. }}$ \\ Tiinamaija Tuomi ${ }^{5,6,7,8}$ • Lisa M. Berglund ${ }^{3,4}$ - Paul W. Franks ${ }^{3,9,10}$ • Jens J. Holst ${ }^{11,12}$ - Rashmi B. Prasad ${ }^{3,4}$. \\ Signe S. Torekov ${ }^{11,12}$. Susana Ravassa ${ }^{13,14,15}$. Javier Díez ${ }^{13,14,15,16,17}$ - Margaretha Persson ${ }^{3}$ Olle Melander $^{3}$. \\ Maria F. Gomez ${ }^{3,4}$. Leif Groop ${ }^{3,4,7}$ • Emma Ahlqvist ${ }^{3,4}$ • Martin Magnusson 1,2,18
}

Received: 24 July 2019 / Accepted: 18 December 2019 / Published online: 23 January 2020

(C) The Author(s) 2020

\begin{abstract}
Aims/hypothesis Evidence that glucose-dependent insulinotropic peptide (GIP) and/or the GIP receptor (GIPR) are involved in cardiovascular biology is emerging. We hypothesised that GIP has untoward effects on cardiovascular biology, in contrast to glucagon-like peptide 1 (GLP-1), and therefore investigated the effects of GIP and GLP-1 concentrations on cardiovascular disease (CVD) and mortality risk.

Methods GIP concentrations were successfully measured during OGTTs in two independent populations (Malmö Diet CancerCardiovascular Cohort [MDC-CC] and Prevalence, Prediction and Prevention of Diabetes in Botnia [PPP-Botnia]) in a total of 8044 subjects. GLP-1 $(n=3625)$ was measured in MDC-CC. The incidence of CVD and mortality was assessed via national/ regional registers or questionnaires. Further, a two-sample Mendelian randomisation (2SMR) analysis between the GIP pathway and outcomes (coronary artery disease [CAD] and myocardial infarction) was carried out using a GIP-associated genetic variant,
\end{abstract}

Emma Ahlqvist and Martin Magnusson contributed equally to this work.

Electronic supplementary material The online version of this article (https://doi.org/10.1007/s00125-020-05093-9) contains peer-reviewed but unedited supplementary material, which is available to authorised users.

Martin Magnusson

martin.magnusson@med.lu.se

1 Department of Clinical Sciences Malmö, Lund University, Clinical Research Centre, Hämtställe HS 36, Box 50332, 202

13 Malmö, Sweden

2 Department of Cardiology, Skåne University Hospital, Inga Marie Nilssons gata 49, 20502 Malmö, Sweden

3 Department of Clinical Sciences Malmö, Lund University, Malmö, Sweden

4 Lund University Diabetes Centre, Lund University, Malmö, Sweden

5 Folkhälsan Research Centre, Biomedicum, Helsinki, Finland

6 Research Program Unit, Diabetes and Obesity, University of Helsinki, Helsinki, Finland

7 Department of Endocrinology, Helsinki University Hospital, Helsinki, Finland

8 Finnish Institute of Molecular Medicine, University of Helsinki, Helsinki, Finland

9 Department of Public Health \& Clinical Medicine, Umeå University, Umeå, Sweden
10 Department of Nutrition, Harvard School of Public Health, Boston, MA, USA

11 Department of Biomedical Sciences, The Panum Institute, University of Copenhagen, Copenhagen, Denmark

12 Novo Nordisk Foundation Center for Basic Metabolic Research, University of Copenhagen, Copenhagen, Denmark

13 Program of Cardiovascular Diseases, CIMA, University of Navarra, Pamplona, Spain

14 CIBERCV, Carlos III Institute of Health, Madrid, Spain

15 Instituto de Investigación Sanitaria de Navarra (IdisNA), Pamplona, Spain

16 Department of Cardiology and Cardiac Surgery, University of Navarra Clinic, Pamplona, Spain

17 Department of Nephrology, University of Navarra Clinic, Pamplona, Spain

18 Wallenberg Center for Molecular Medicine, Lund University, Malmö, Sweden 


\section{Research in context}

\section{What is already known about this subject?}

- Glucagon-like peptide 1 (GLP-1) analogue therapy has proven to be beneficial for cardiovascular protection. On the contrary, evidence that glucose-dependent insulinotropic peptide (GIP) and/or the GIP receptor (GIPR) have untoward effects on cardiovascular biology is emerging

- Currently, prospective data on the incretin system's long-term effects on mortality and cardiovascular disease are sparse

What is the key question?

- Do GIP levels have untoward effects on cardiovascular disease and mortality risk, in contrast to GLP-1 levels?

What are the new findings?

- In two prospective, community-based studies, elevated levels of GIP were associated with greater risk of cardiovascular and total mortality, within 5-9 years of follow-up

- Further, a bi-directional two-sample Mendelian randomisation analysis provides novel evidence of GIP's involvement in cardiovascular disease

- Physiological GLP-1 levels did not significantly influence the risk of mortality

How might this impact on clinical practice in the foreseeable future?

- Our findings, showing that physiological increased levels of GIP are associated with cardiovascular and all-cause mortality, could motivate more thorough investigation of potential risks associated with pharmacological stimulation of the GIP pathway

rs1800437, as instrumental variable. An additional reverse 2SMR was performed with CAD as exposure variable and GIP as outcome variable, with the instrumental variables constructed from 114 known genetic risk variants for CAD.

Results In meta-analyses, higher fasting levels of GIP were associated with risk of higher total mortality $(\mathrm{HR}[95 \% \mathrm{CI}]=1.22$ $\left.[1.11,1.35] ; p=4.5 \times 10^{-5}\right)$ and death from CVD $(\mathrm{HR}[95 \% \mathrm{CI}] 1.30[1.11,1.52] ; p=0.001)$. In accordance, 2SMR analysis revealed that increasing GIP concentrations were associated with CAD and myocardial infarction, and an additional reverse 2SMR revealed no significant effect of CAD on GIP levels, thus confirming a possible effect solely of GIP on CAD.

Conclusions/interpretation In two prospective, community-based studies, elevated levels of GIP were associated with greater risk of all-cause and cardiovascular mortality within 5-9 years of follow-up, whereas GLP-1 levels were not associated with excess risk. Further studies are warranted to determine the cardiovascular effects of GIP per se.

Keywords Cardiovascular · Cardiovascular events · Coronary artery disease · GIP · GLP-1 · Glucagon-like peptide 1 . Glucose-dependent insulinotropic peptide $\cdot$ Mendelian randomisation $\cdot$ Mortality

\section{Abbreviations}

CAD

CVD

FPG

GIP

GIPR

GLP-1

IVW

MDC-CC

MR

OPN

PPP-Botnia
Coronary artery disease

Cardiovascular disease

Fasting plasma glucose

Glucose-dependent insulinotropic polypeptide

GIP receptor

Glucagon-like peptide-1

Inverse variance weighted method

Malmö Diet and

Cancer-Cardiovascular Cohort

Mendelian randomisation

Osteopontin

Prevalence, Prediction and Prevention

of Diabetes in Botnia
SBP

2SMR

Systolic BP

Two-sample Mendelian randomisation

\section{Introduction}

The enteroendocrine peptide glucose-dependent insulinotropic polypeptide (GIP) and proglucagon-derived peptides, such as glucagon-like peptide-1 (GLP-1), were classically viewed as regulators of islet function, nutrient absorption, appetite and energy homeostasis [1]. The observation that the G-proteincoupled receptors, through which these regulatory peptides exert their effects, are widely expressed in the cardiovascular 
system has triggered a lot of interest in their translational relevance beyond metabolic control [2].

Both experimental and clinical data, such as the outcomes from the LEADER, SUSTAIN-6, HARMONY and REWIND trials, support therapeutic benefits of GLP-1 receptor agonists with regards to cardiovascular outcomes in type 2 diabetes [3-7]. Further, a missense variant in the gene encoding the GLP-1 receptor has been associated with protection against heart disease [8]. While the bulk of the studies published so far have focused on GLP-1, GIP has received less attention. Data from our laboratory demonstrated that fasting GIP concentrations were significantly higher in individuals with a history of cardiovascular disease (CVD) than in those without, and that GIP receptor (GIPR) gene mRNA expression is higher in the arterial wall of individuals with symptoms of CVD [9]. Moreover, a common variant in GIPR (rs10423928), which is in complete linkage disequilibrium with rs1800437, associates with increased risk of stroke in individuals with type 2 diabetes and, recently, Ussher et al. demonstrated that reduction in GIPR signalling is linked to ischaemic cardioprotection in mice [10]. Thus, evidence that GIP and/or GIPR are involved in cardiovascular biology is emerging. In light of these findings, we explored whether circulating levels of GIP (and GLP-1) are associated with cardiovascular death and total mortality risk in two large, population-based cohorts. We also performed a two-sample Mendelian randomisation (2SMR) analysis using the GIPR variant rs 1800437 previously associated with features of the metabolic syndrome and CVD [11] as an instrumental variable to study the effect of increased GIP levels on coronary artery disease (CAD) and myocardial infarction. Furthermore, a 2SMR analysis in a reverse direction from CAD to GIP was performed, using 114 known genetic risk variants for $\mathrm{CAD}$ as instrumental variables.

\section{Methods}

\section{Prevalence, Prediction and Prevention of Diabetes in Botnia study}

The Prevalence, Prediction and Prevention of DiabetesBotnia (PPP-Botnia) study is a population-based study in western Finland started in 2004 to obtain estimates of prevalence and risk factors for type 2 diabetes, impaired glucose tolerance, impaired fasting glucose and the metabolic syndrome in the adult population. Participants were randomly recruited from the national Finnish Population Registry to represent $6-7 \%$ of the population in the 18-75 year age range (mean age $51 \pm 17$ years) [12]. Altogether, 5208 individuals participated in the study ( $54.7 \%$ of those invited). A follow-up study was conducted between 2011 and 2015, in which 3870 $(74.3 \%)$ individuals participated. After exclusion of individuals with partially missing data, 4572 individuals remained for analysis of fasting GIP and 4398 for post-challenge GIP (see electronic supplementary material [ESM] Fig. 1). The number of individuals with diabetes included in analysis was 307 at the basal visit and 284 at the re-investigation visit. Diagnosis of diabetes was confirmed from participants' records or based on fasting plasma glucose concentration $\geq 7.0 \mathrm{mmol} / 1$ and/or post-challenge glucose $\geq 11.1 \mathrm{mmol} / \mathrm{l}$. The participants gave their written informed consent and the study protocol was approved by the Ethics Committee of Helsinki University Hospital, Finland.

\section{Malmö Diet and Cancer-Cardiovascular Cohort, Sweden}

Between 1991 and 1996, a prospective, population-based study, the Malmö Diet and Cancer study, was conducted in the city of Malmö, Sweden, including questionnaires, blood sample donations and anthropometrical measurements at the baseline examination $(n=30,447)$. All people born in the years 1926-1945 and living in Malmö were invited to participate. To study cardiovascular risk factors, a sample of the study population $(n=6103)$ was randomised into a substudy, the Malmö Diet and CancerCardiovascular Cohort (MDC-CC) [13]. During 2007-2012, a new clinical examination was performed $(n=3734)$ within the MDC-CC, with the addition of OGTT [14]. A schematic description of the study population is presented in ESM Fig. 2. Fasting blood samples were collected from 3692 individuals (fasting GIP available in $n=3479$ ). Four-hundred-and-forty-nine individuals did not perform the complete OGTT (386 with previously known diabetes, 63 for various reasons), resulting in postchallenge $(2 \mathrm{~h})$ blood samples available in 3243 individuals (post-challenge GIP available in $n=3070$ ). The characteristics of non-attendees at the re-examination have been described elsewhere [13]. The participants gave their written informed consent and the study protocol was approved by the Ethical Review Board, Lund, Sweden.

\section{Genotyping}

In both cohorts, information on genotype rs1800437 was obtained from genome-wide association study data performed at the Broad genotyping facility using Illumina OmniExpressExome BeadChip v1.0 B (MDC-CC, $n=3344$ ) or Illumina HumanExome BeadChip v1.0 (PPP-Botnia, $n=$ 4905). The call rate was $>99.9 \%$ and the SNP was in HardyWeinberg equilibrium in both cohorts.

\section{Clinical assessment}

PPP-Botnia Two BP recordings were obtained from the right arm of a sitting person after $30 \mathrm{~min}$ of rest and their mean value was calculated. If there was more than $5 \mathrm{mmHg}$ difference between 
Table 1 Characteristics of the study population within quartiles of GIP plasma concentrations in PPP-Botnia

\begin{tabular}{|c|c|c|c|c|c|}
\hline Characteristic & Total & Q1 & Q2 & Q3 & Q4 \\
\hline \multicolumn{6}{|l|}{ Fasting GIP } \\
\hline No. of participants & 4572 & 1128 & 1163 & 1157 & 1124 \\
\hline Age, years & $49.7 \pm 15.8$ & $48.0 \pm 15.6$ & $49.5 \pm 15.7$ & $50.9 \pm 15.9$ & $50.4 \pm 15.7$ \\
\hline Female sex & $2421(53.0)$ & $650(57.6)$ & $607(52.2)$ & $615(53.3)$ & $549(48.8)$ \\
\hline BMI, $\mathrm{kg} / \mathrm{m}^{2}$ & $26.5 \pm 4.4$ & $26.1 \pm 4.5$ & $26.4 \pm 4.1$ & $26.5 \pm 4.3$ & $27.0 \pm 4.8$ \\
\hline Fasting GIP, pmol/1 & $31.7(21.7-46.1)$ & $16.6(13.4-19.2)$ & $26.7(24.2-29.3)$ & $37.9(34.8-41.8)$ & $61.4(52.8-77.1)$ \\
\hline Fasting insulin, pmol/1 & $38.2(26.1-56.5)$ & $36.3(25.1-52.7)$ & $36.1(25.8-53.1)$ & $38.5(25.8-57.4)$ & $42.8(28.3-68.3)$ \\
\hline $\mathrm{FPG}, \mathrm{mmol} / \mathrm{l}$ & $5.4 \pm 0.9$ & $5.4 \pm 0.7$ & $5.4 \pm 0.8$ & $5.4 \pm 0.8$ & $5.5 \pm 1.4$ \\
\hline $\mathrm{SBP}, \mathrm{mmHg}$ & $133.6 \pm 19.3$ & $131.4 \pm 18.5$ & $133.0 \pm 18.7$ & $134.6 \pm 20.3$ & $135.3 \pm 19.6$ \\
\hline LDL-cholesterol, mmol/1 & $3.3(2.6-3.9)$ & $3.2(2.6-3.8)$ & $3.2(2.6-3.9)$ & $3.3(2.7-4.0)$ & $3.3(2.7-3.9)$ \\
\hline HDL-cholesterol, mmol/1 & $1.37(1.13-1.65)$ & $1.40(1.16-1.66)$ & $1.40(1.14-1.68)$ & $1.37(1.13-1.65)$ & $1.33(1.10-1.62)$ \\
\hline Diabetes & $272(5.9)$ & $37(3.3)$ & $50(4.3)$ & $70(6.1)$ & $115(10.3)$ \\
\hline Number of events (total mortality) & $154(3.0)$ & 26 & 36 & 36 & 56 \\
\hline GIPR rs 1800437, MAF & 0.28 & 0.33 & 0.27 & 0.27 & 0.24 \\
\hline \multicolumn{6}{|l|}{ Post-challenge GIP } \\
\hline No. of participants & 4398 & 1034 & 1104 & 1131 & 1129 \\
\hline Age, years & $49.6 \pm 15.7$ & $45.6 \pm 15.1$ & $47.0 \pm 15.7$ & $50.3 \pm 15.3$ & $55.0 \pm 15.0$ \\
\hline Female sex & $2326(52.9)$ & $414(40.0)$ & $518(46.9)$ & $625(55.3)$ & $769(68.1)$ \\
\hline BMI, $\mathrm{kg} / \mathrm{m}^{2}$ & $26.4 \pm 4.4$ & $26.7 \pm 4.7$ & $26.4 \pm 4.4$ & $26.3 \pm 4.3$ & $26.2 \pm 4.1$ \\
\hline Post-challenge GIP, pmol/1 & $178.3(131.5-237.4)$ & $100.1(82.3-114.3)$ & $153.5(140.7-163.4)$ & $202.9(188.7-217.8)$ & $294.1(259.7-349.5)$ \\
\hline Post-challenge insulin, pmol/1 & $168.8(100.0-284.0)$ & $126.4(63.2-222.9)$ & $156.9(93.1-267.4)$ & $183.3(111.8-297.9)$ & $200.0(129.9-327.0)$ \\
\hline Post-challenge glucose, $\mathrm{mmol} / \mathrm{l}$ & $5.5 \pm 2.2$ & $5.3 \pm 2.5$ & $5.5 \pm 2.2$ & $5.4 \pm 1.9$ & $5.8 \pm 2.3$ \\
\hline $\mathrm{SBP}, \mathrm{mmHg}$ & $133.3 \pm 19.0$ & $132.4 \pm 18.5$ & $131.5 \pm 18.1$ & $134.0 \pm 19.6$ & $135.1 \pm 19.6$ \\
\hline LDL-cholesterol, mmol/1 & $3.3(2.6-3.9)$ & $3.2(2.5-3.7)$ & $3.2(2.6-3.8)$ & $3.4(2.8-4.0)$ & $3.5(2.8-4.2)$ \\
\hline HDL-cholesterol, mmol/1 & $1.38(1.14-1.66)$ & $1.35(1.12-1.60)$ & $1.36(1.13-1.64)$ & $1.36(1.13-1.63)$ & $1.43(1.18-1.73)$ \\
\hline Diabetes & $194(4.4)$ & $61(5.9)$ & $40(3.6)$ & $37(3.3)$ & $56(5.0)$ \\
\hline No. of events (total mortality) & 130 & 26 & 24 & 32 & 48 \\
\hline GIPR rs1800437, MAF & 0.28 & 0.32 & 0.28 & 0.27 & 0.24 \\
\hline
\end{tabular}

Values are expressed as mean $\pm \mathrm{SD}$, median (25th-75th interquartile range) or $n(\%)$

MAF, minor allele frequency; Q1, quartile with the lowest values; Q4, quartile with the highest values

the two recordings, the recording was repeated. BMI was calculated as weight $(\mathrm{kg})$ divided by the square of the height $(\mathrm{m})$.

MDC-CC BP was obtained after $10 \mathrm{~min}$ of rest in the supine position. BMI was calculated as weight $(\mathrm{kg})$ divided by the square of the height (m).

OGTT In both cohorts, a $75 \mathrm{~g}$ OGTT, the most appropriate method for the clinical assessment of glucometabolic status [14], was performed after an overnight fast. The OGTT was performed according to same standardised protocol in both cohorts (individuals with known diabetes did not undergo an OGTT).

\section{Laboratory assays}

For both PPP-Botnia and MDC-CC participants, GIP was analysed by the same laboratory using the following procedure: during OGTT, blood samples were drawn in order to analyse GIP at 0 and $120 \mathrm{~min}$. Serum GIP was analysed using Millipore's Human GIP Total ELISA (Merck Millipore, Darmstadt, Germany; no. EZHGIP-54 K; minimum detection level $1.65 \mathrm{pmol} / \mathrm{l}$, intra- and inter-assay CV 1.8-6.1\% and 3$8.8 \%$, respectively) [15].

PPP-Botnia Serum insulin was measured by an AutoDelfia fluoroimmunometric assay (PerkinElmer, Waltham, MA, USA). Fasting plasma glucose was analysed using the HemoCue Glucose System (HemoCue, Ängelholm, Sweden). Serum total cholesterol, HDL-cholesterol and triacylglycerol concentrations were measured first on a Cobas Mira analyser (Hoffman-La Roche, Basel, Switzerland) and LDL-cholesterol concentrations were calculated using the Friedewald formula. Since January 2006, total cholesterol, LDL-cholesterol, HDLcholesterol and triacylglycerol concentrations have been measured using an enzymatic method (Konelab 60i analyser; Thermo Electron Oy, Vantaa, Finland). 
Table 2 Characteristics of the study population within quartiles of GIP plasma concentrations in MDC-CC

\begin{tabular}{|c|c|c|c|c|c|}
\hline Characteristic & Total & Q1 & Q2 & Q3 & Q4 \\
\hline No. of participants & 3479 & 871 & 870 & 867 & 871 \\
\hline \multicolumn{6}{|l|}{ Fasting GIP } \\
\hline Age, years & $72.4 \pm 5.6$ & $71.6 \pm 5.4$ & $72.5 \pm 5.6$ & $72.8 \pm 5.6$ & $72.9 \pm 5.6$ \\
\hline Female sex & $2184(59.1)$ & $523(60)$ & $503(57.9)$ & $494(57.1)$ & $522(59.9)$ \\
\hline BMI, $\mathrm{kg} / \mathrm{m}^{2}$ & $26.9 \pm 4.4$ & $26.4 \pm 3.9$ & $26.6 \pm 4.0$ & $26.8 \pm 4.4$ & $27.8 \pm 5.0$ \\
\hline Fasting GIP, pmol/1 & $41.2(30.4-56.8)$ & $24.5(20.3-27.6)$ & $35.9(33.2-38.3)$ & $47.6(44.4-51.5)$ & $72.9(63.3-89.3)$ \\
\hline Fasting insulin, $\mathrm{pmol} / 1$ & $53.5(37.5-77.1)$ & $47.9(34.7-66.0)$ & $50.0(35.4-72.2)$ & $54.2(38.9-77.1)$ & $65.3(44.4-93.1)$ \\
\hline $\mathrm{FPG}, \mathrm{mmol} / 1$ & $5.9(5.4-6.5)$ & $5.8(5.3-6.3)$ & $5.8(5.4-6.4)$ & $5.9(5.4-6.5)$ & $6.1(5.5-6.8)$ \\
\hline $\mathrm{SBP}, \mathrm{mmHg}$ & $143 \pm 18$ & $141 \pm 17$ & $144 \pm 19$ & $144 \pm 18$ & $144 \pm 20$ \\
\hline LDL-cholesterol, mmol/1 & $3.3(2.6-3.9)$ & $3.4(2.8-4.0)$ & $3.3(2.7-3.9)$ & $3.2(2.6-3.9)$ & $3.1(2.4-3.8)$ \\
\hline HDL-cholesterol, mmol/1 & $1.4(1.1-1.7)$ & $1.4(1.1-1.7)$ & $1.4(1.1-1.7)$ & $1.4(1.1-1.7)$ & $1.3(1.0-1.6)$ \\
\hline Diabetes & $170(4.9)$ & $37(4.3)$ & $42(4.8)$ & $45(5.1)$ & $46(5.2)$ \\
\hline No. of events (total mortality) & $346(10.0)$ & $60(6.9)$ & $74(8.5)$ & $88(10.2)$ & $124(14.2)$ \\
\hline GIPR rs 1800437, MAF & 0.22 & 0.27 & 0.21 & 0.21 & 0.20 \\
\hline \multicolumn{6}{|l|}{ Post-challenge GIP } \\
\hline No. of participants & 3070 & 768 & 766 & 769 & 767 \\
\hline Age, years & $72.4 \pm 5.6$ & $71.1 \pm 5.6$ & $72.0 \pm 5.3$ & $72.8 \pm 5.6$ & $73.7 \pm 5.5$ \\
\hline Female sex & $1830(59.6)$ & $355(46.2)$ & $425(55.5)$ & $488(63.5)$ & $562(73.3)$ \\
\hline BMI, $\mathrm{kg} / \mathrm{m}^{2}$ & $26.6 \pm 4.2$ & $27.1 \pm 4.2$ & $26.4 \pm 3.9$ & $26.5 \pm 4.0$ & $26.4 \pm 4.4$ \\
\hline Post-challenge GIP, pmol/1 & $222.7(163.2-293.8)$ & $129.7(106.4-147.1)$ & $193.0(178.1-207.0)$ & $253.7(237.3-272.4)$ & $356.8(321.0-414.1)$ \\
\hline Post-challenge insulin, pmol/1 & $277.0(179.9-441.0)$ & $257.6(171.5-400.7)$ & $266.7(166.0-427.8)$ & $273.6(189.6-441.0)$ & $311.1(191.7-520.1)$ \\
\hline Post-challenge glucose, $\mathrm{mmol} / \mathrm{l}$ & $6.8(5.5-8.2)$ & $6.6(5.5-8.1)$ & $6.7(5.4-8.1)$ & $6.8(5.5-8.32)$ & $7.0(5.6-8.8)$ \\
\hline $\mathrm{SBP}, \mathrm{mmHg}$ & $143 \pm 19$ & $143 \pm 19$ & $144 \pm 19$ & $143 \pm 19$ & $143 \pm 19$ \\
\hline LDL-cholesterol, mmol/1 & $3.3(2.7-4.0)$ & $3.3(2.7-3.9)$ & $3.4(2.7-4.0)$ & $3.4(2.7-4.0)$ & $3.3(2.7-4.0)$ \\
\hline HDL-cholesterol, mmol/1 & $1.4(1.1-1.7)$ & $1.4(1.0-1.7)$ & $1.4(1.1-1.7)$ & $1.4(1.1-1.7)$ & $1.4(1.2-1.7)$ \\
\hline Diabetes & $165(5.4)$ & $47(6.2)$ & $38(4.9)$ & $34(4.4)$ & $46(5.9)$ \\
\hline No. of events (total mortality) & $282(9.2)$ & $49(6.4)$ & $66(8.6)$ & $60(7.8)$ & $104(13.6)$ \\
\hline GIPR rs 1800437, MAF & 0.22 & 0.26 & 0.23 & 0.21 & 0.18 \\
\hline
\end{tabular}

Values are expressed as mean $\pm \mathrm{SD}$, median (25th-75th interquartile range) or $n(\%)$

MAF, minor allele frequency; Q1, quartile with the lowest values; Q4, quartile with the highest values

MDC-CC During OGTT, blood samples were drawn in order to analyse GLP-1 at 0 and $120 \mathrm{~min}$. Total plasma GLP-1 concentrations (intact GLP-1 and the metabolite GLP-1 9-36-amide) were determined radioimmunologically (minimum detection limit 1 pmol/1; intra- and inter-assay $\mathrm{CV}<6.0 \%$ and $<15 \%$, respectively). Identical quality controls and batches for all reagents in each analysis set were used in a consecutive sample analysis during 2 months [15]. Fasting plasma glucose was analysed using the HemoCue Glucose System. Serum insulin was assayed with Dako ELISA kit (K6219, Dako, Stockholm, Sweden; minimum detection level $3 \mathrm{pmol} / \mathrm{l}$, intra- and inter-assay CV 5.1-7.5\% and 4.2-9.3\%, respectively) at the Department of Clinical Chemistry, Malmö University Hospital. HDL-cholesterol was analysed according to standard procedures at the Department of Clinical Chemistry, University Hospital Malmö. LDL-cholesterol was calculated according to the Friedewald formula.

\section{Classification of endpoints}

PPP-Botnia Mortality data were obtained from death certificates through the national registry for Causes of Death (Statistics Finland) until the end of 2014. Endpoints were defined on the basis of ICD10 codes (http://apps.who.int/ classifications/icd10/browse/2016/en). Incidence and prevalence of CVD was based on a questionnaire completed by the participants at the basal and follow-up visits (for questionnaire and definitions, see ESM Methods).

Death from myocardial infarction was defined by ICD10 codes I212, I214, I219 and stroke by ICD10 codes I601, I610, I619, I620, I630, I634, I635 and I639. Death from CVD was defined by code groups I110, I119, I120, I212, I214, I219, I250, I251, I258, I259, I260, I269, I350, I420, I48, I601, I610, I619, I620, I630, I634, I635, I639, I713 and I693. 
Table 3 Associations of $1 \mathrm{SD}$ of log-transformed fasting GIP and post-challenge GIP with total and cardiovascular mortality risk

\begin{tabular}{|c|c|c|c|c|c|c|}
\hline \multirow[t]{2}{*}{ Variable } & \multicolumn{2}{|l|}{ PPP-Botnia } & \multicolumn{2}{|l|}{ MDC-CC } & \multicolumn{2}{|l|}{ Meta-analysis } \\
\hline & HR $(95 \%$ CI) & $p$ value & HR $(95 \%$ CI) & $p$ value & HR $(95 \%$ CI $)$ & $p$ value \\
\hline \multicolumn{7}{|l|}{ Fasting GIP } \\
\hline \multicolumn{7}{|c|}{ Total mortality } \\
\hline Model $1^{\mathrm{a}}$ & $1.29(1.10,1.50)$ & 0.001 & $1.27(1.14,1.40)$ & $6.0 \times 10^{-6}$ & $1.28(1.17,1.39)$ & $4.7 \times 10^{-8}$ \\
\hline Model $2^{\mathrm{b}}$ & $1.19(1.02,1.40)$ & 0.029 & $1.26(1.13,1.40)$ & $4.9 \times 10^{-5}$ & $1.24(1.13,1.35)$ & $3.0 \times 10^{-6}$ \\
\hline Model $3^{\mathrm{c}}$ & $1.21(1.03,1.43)$ & 0.025 & $1.23(1.09,1.37)$ & $4.3 \times 10^{-4}$ & $1.22(1.11,1.35)$ & $4.5 \times 10^{-5}$ \\
\hline \multicolumn{7}{|c|}{ Cardiovascular mortality } \\
\hline Model $1^{\mathrm{a}}$ & $1.42(1.11,1.83)$ & 0.007 & $1.24(1.04,1.48)$ & 0.019 & $1.29(1.12,1.50)$ & $5.0 \times 10^{-4}$ \\
\hline Model $2^{\mathrm{b}}$ & $1.34(1.02,1.76)$ & 0.033 & $1.23(1.02,1.48)$ & 0.029 & $1.26(1.08,1.48)$ & 0.0029 \\
\hline Model $3^{\mathrm{c}}$ & $1.41(1.07,1.85)$ & 0.015 & $1.25(1.03,1.51)$ & 0.023 & $1.30(1.11,1.52)$ & 0.0012 \\
\hline \multicolumn{7}{|c|}{ Post-challenge GIP } \\
\hline \multicolumn{7}{|c|}{ Total mortality } \\
\hline Model $1^{\mathrm{a}}$ & $0.97(0.81,1.17)$ & 0.755 & $1.24(1.09,1.40)$ & 0.001 & $1.15(1.03,1.28)$ & 0.01 \\
\hline Model $2^{\mathrm{b}}$ & $1.00(0.83,1.20)$ & 0.960 & $1.27(1.11,1.45)$ & 0.001 & $1.18(1.06,1.32)$ & 0.004 \\
\hline Model $3^{\mathrm{c}}$ & $1.00(0.83,1.22)$ & 0.978 & $1.23(1.07,1.41)$ & 0.003 & $1.14(1.02,1.28)$ & 0.02 \\
\hline \multicolumn{7}{|c|}{ Cardiovascular mortality } \\
\hline Model $1^{\mathrm{a}}$ & $1.05(0.76,1.44)$ & 0.771 & $1.50(1.21,1.85)$ & $2.4 \times 10^{-5}$ & $1.32(1.10,1.57)$ & 0.0023 \\
\hline Model $2^{\mathrm{b}}$ & $1.10(0.80,1.53)$ & 0.556 & $1.56(1.24,1.96)$ & $1.7 \times 10^{-4}$ & $1.39(1.16,1.63)$ & $5.0 \times 10^{-4}$ \\
\hline Model $3^{\mathrm{c}}$ & $1.10(0.79,1.52)$ & 0.578 & $1.51(1.20,1.91)$ & $4.9 \times 10^{-4}$ & $1.36(1.13,1.65)$ & 0.0013 \\
\hline
\end{tabular}

No. of individuals (events) included in analyses for fasting GIP: total mortality in PPP-Botnia $n=4572$ (154), in MDC-CC $n=3472$ (346) and in metaanalysis $n=8044$ (500); cardiovascular mortality in PPP-Botnia $n=4571$ (53), in MDC $n=3472$ (120) and in meta-analysis $n=8043$ (173). No. of individuals (events) included in analyses for post-challenge GIP: total mortality in PPP-Botnia $n=4398$ (130), in MDC-CC $n=3060$ (279) and in metaanalysis: $n=7458$ (409); cardiovascular mortality in PPP-Botnia $n=4398$ (46), in MDC-CC $n=2827$ (89) and in meta-analyses $n=7225$ (135)

${ }^{\text {a }}$ Model 1 is adjusted for age and sex

${ }^{\mathrm{b}}$ Model 2 is adjusted for age, sex, BMI, SBP, fasting or post-challenge glucose, fasting or post-challenge insulin, LDL-cholesterol, HDL-cholesterol and smoking

${ }^{\mathrm{c}}$ Model 3 is adjusted for lipid-lowering treatment, BP-lowering treatment, diabetes status and educational level on top of covariates in Model 2

MDC-CC Swedish personal identification numbers were linked to national registers (The Swedish Hospital Discharge Register and The Swedish Cause of Death Register; The National Board of Health and Welfare) [16] for cardiovascular endpoint retrieval until the end of 2014. All cardiovascular endpoints were defined on the basis of ICD8 (http://www. wolfbane.com/icd/icd8.htm), ICD9 (www.icd9data.com/ 2007/Volume1) and ICD10 codes. Coronary events were defined as acute myocardial infarction, other acute and subacute forms of ischaemic heart disease, old myocardial

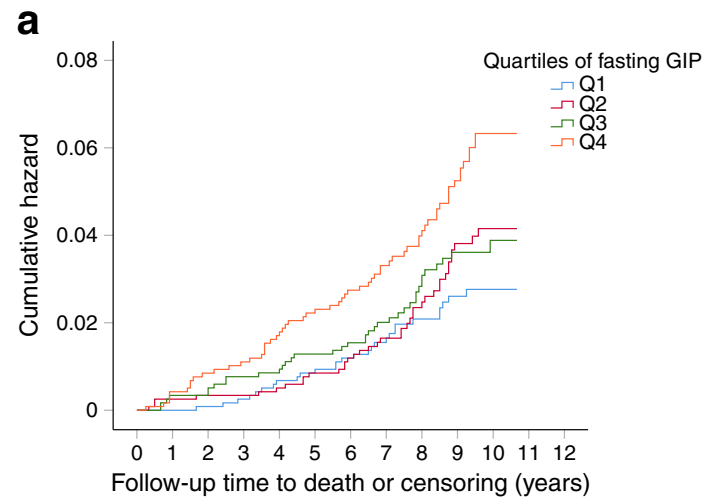

Fig. 1 Total mortality risk in quartiles of fasting GIP. (a) Cumulative hazard for total mortality over a mean follow-up of 8.8 years for fasting GIP quartiles in PPP-Botnia $(p=0.001)$. (b) Cumulative hazard for total

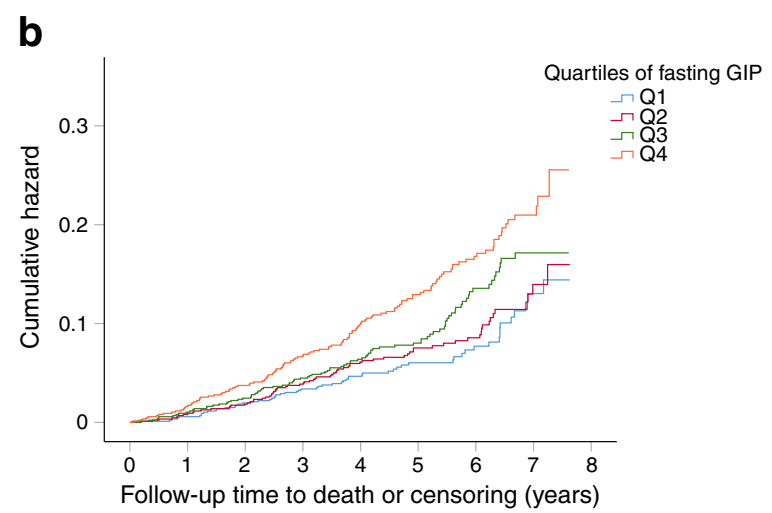

mortality over a mean follow-up of 5.1 years for fasting GIP quartiles $\left(p=3 \times 10^{-5}\right)$ in MDC-CC. Q1, quartile with the lowest values; Q4, quartile with the highest values 
Table 4 Associations of 1 SD of log-transformed fasting GIP and postchallenge GIP with incident non-fatal cardiovascular events

\begin{tabular}{llllll}
\hline Variable & \multicolumn{2}{l}{ PPP-Botnia } & & & \multicolumn{2}{l}{ MDC-CC } \\
\cline { 2 - 3 } \cline { 5 - 6 } \cline { 5 - 6 } & OR $(95 \% \mathrm{CI})$ & $p$ value & & HR $(95 \% \mathrm{CI})$ & $p$ value \\
\hline Fasting GIP & & & & \\
Model 1 $^{\mathrm{a}}$ & $1.33(1.10,1.60)$ & 0.003 & & $1.13(0.98,1.31)$ & 0.088 \\
Model 2 $^{\mathrm{b}}$ & $1.26(1.04,1.52)$ & 0.019 & & $1.06(0.92,1.23)$ & 0.406 \\
Model 3 $^{\mathrm{c}}$ & $1.25(1.03,1.52)$ & 0.024 & & $1.07(0.93,1.24)$ & 0.362 \\
Post-challenge GIP & & & 0.758 \\
Model 1 $^{\mathrm{a}}$ & $1.20(0.98,1.47)$ & 0.073 & & $0.98(0.83,1.14)$ & 0.758 \\
Model 2 $^{\mathrm{b}}$ & $1.24(1.01,1.53)$ & 0.040 & & $0.97(0.82,1.15)$ & 0.748 \\
Model 3 $^{\mathrm{c}}$ & $1.25(1.02,1.54)$ & 0.035 & & $0.97(0.82,1.15)$ & 0.708 \\
\hline
\end{tabular}

No. of individuals and incident non-fatal cardiovascular events included in analyses: for PPP-Botnia $n=3084$ (128) and for MDC $n=2708$ (202). The two cohorts were analysed with different methods (logistic regression in PPP-Botnia, Cox regression in MDC-CC), since exact time to event was not known for PPP-Botnia. Because of this, and because endpoints were defined and recorded differently, no meta-analysis was performed

${ }^{a}$ Model 1 is adjusted for age and sex

${ }^{\mathrm{b}}$ Model 2 is adjusted for age, sex, BMI, SBP, fasting or post-challenge glucose, fasting or post-challenge insulin, LDL-cholesterol, HDL-cholesterol and smoking

${ }^{\mathrm{c}}$ Model 3 is adjusted for lipid-lowering treatment, BP-lowering treatment, diabetes status and educational level on top of covariates in Model 2

infarction, angina pectoris or other forms of chronic ischaemic heart disease, and identified using codes 410-414 (ICD8), 410-414 (ICD9) and I21, I252, I20, I251, I253-I259 (ICD10). Angioplasty events were obtained from the Swedish Coronary Angiography and Angioplasty Register (SCAAR, Kranskärlsregistret) from Uppsala Clinical Research Center, Akademiska sjukhuset, Uppsala, Sweden. Coronary artery bypass grafting was identified through original surgery codes of the first incident surgery event. The following surgery codes were extracted: Op6 (1964-96): 3065, 3066, 3068, 3080, 3092, 3105, 3127, 3158 KKÅ97 (1997-) (including subgroups). Fatal or non-fatal stroke was defined as subarachnoid haemorrhage, intracerebral haemorrhage, occlusion of cerebral arteries, acute (but ill-defined) cerebrovascular disease or stroke of unknown origin and identified using codes 430, 431, 434 and 436 (ICD9) and I60, I61, I63 and I64 (ICD10). Stroke events were extracted from Stromaregistret and Recidivregistret at the Cardiovascular epidemiology research group, SUS Malmö, Sweden. Heart failure was retrieved through codes 427.00, 427.10 and 428 . 99 (ICD8), 428 (ICD9) and I50 and I11.0 (ICD10). All-cause death (or otherwise emigration for censored cases) was identified through Swedish total population register Statistics Sweden, The Swedish Tax Agency and The National Board of Health and Welfare. Death from CVD was identified through codes ICD9:390-459 and ICD10:I [16-19].

\section{Statistical analysis}

All analyses were performed in SPSS v.22.0 (SPSS, Armonk, NY, USA), except for the Mendelian randomisation (MR) analyses and the meta-analyses, which were performed using $\mathrm{R}$ software version 3.5.2 [20]. The 2SMR analyses were built using MendelianRandomization [21] and TwoSampleMR [22] packages. A two-tailed $p$ value $<0.05$ was considered significant. Skewed continuous variables were logarithmically transformed. Individuals with missing values on covariates were excluded from respective analysis.

Cox regression models were used to calculate HRs for each 1 SD increment of log-transformed fasting and post-challenge GIP and GLP-1 concentrations on mortality from CVD and total mortality risk. Individuals who died from external causes were censored.

Table 5 MR analyses

\begin{tabular}{lllllll}
\hline Exposure & Outcome & Method & IV & $\beta$ & SE & $p$ value \\
\hline Fasting GIP & CAD & Wald ratio (2SMR) & rs1800437 & 0.51 & 0.165 & 0.002 \\
Fasting GIP & MI & Wald ratio (2SMR) & rs1800437 & 0.46 & 0.186 & 0.013 \\
Fasting GIP $^{\mathrm{b}}$ & CAD & Wald ratio (2SMR) & rs1800437 & 0.42 & 0.129 & 0.001 \\
CAD $^{\mathrm{c}}$ & Fasting GIP & IVW & 114 SNPs & -0.042 & 0.029 & 0.148 \\
CAD $^{\mathrm{c}}$ & Fasting GIP & MR Egger & 114 SNPs & -0.039 & 0.074 & 0.595 \\
\hline
\end{tabular}

${ }^{\text {a }}$ Data from CARDIoGRAMplusC4D for CAD ( $n=184,305$; 60,801 cases, 123,504 controls) and myocardial infarction ( $n=171,875 ; 43,676$ cases, 128,199 controls)

${ }^{\mathrm{b}}$ Data for CAD from UK Biobank ( $n=296,525 ; 34,541$ cases, 61,984 controls)

${ }^{\mathrm{c}}$ Loci from CARDiOGRAMplusC4D and UK Biobank were used for constructing the instrumental variable. The summary data for the outcome (fasting GIP) was acquired from the MDC-CC cohort. Out of 147 SNPs in CARDiOGRAMplusC4D and UK Biobank (ESM Table 1) with $p<5 \times 10^{-8}$ and $r^{2}$ measure of linkage disequilibrium $<0.2,116$ SNPs were selected with available information in MDC-CC (ESM Table 2). An additional two SNPs (rs472109, rs4754698) were removed from analysis for being palindromic with intermediate allele frequencies

IV, instrumental variable; MI, myocardial infarction 
As for analyses of incident non-fatal CVD, the two cohorts were analysed with different methods (Cox regression in MDC-CC, logistic regression in PPP-Botnia), since exact time to event was not known for PPP-Botnia. Because of this, and because endpoints were defined and recorded differently, no meta-analysis was performed. Further, in exploratory, crosssectional analyses for associations between GIP concentration and prevalent subtypes of CVD, logistic regression was used to calculate ORs. Model 1 (adjusted for age and sex) was used for the primary analysis and further adjusted for relevant physiological covariates in Model 2 (BMI, systolic BP [SBP], fasting plasma glucose [FPG], fasting insulin, LDL-cholesterol, HDLcholesterol and smoking for analyses of fasting GIP, and BMI, SBP, post-challenge glucose, post-challenge insulin, LDLcholesterol, HDL-cholesterol and smoking for analyses of post-challenge GIP). Further adjustment on top of Model 2 was carried out by entering diabetes status, lipid-lowering treatment (LLT), BP-lowering treatment and educational level into Model 3. Proportional hazard assumptions were tested using Schoenfeld residuals. Fixed-effects meta-analysis of mortality variables was performed in $\mathrm{R}$ using the metafor package [23].

A 2SMR was performed with fasting GIP levels as exposure variable, $\mathrm{CAD}$ and myocardial infarction were defined as outcome variables, and rs1800437 as the instrumental variable. We applied the Wald ratio method as statistical modelling for the 2SMR analysis with a summary data for the outcomes from CARDIoGRAMplusC4D consortium and UK Biobank. In addition, to further explore the direction of association between GIP and CAD, we carried out a reverse $2 \mathrm{SMR}$ analysis from CAD to fasting GIP. Loci from a meta-analysis of CARDiOGRAMplusC4D and UK Biobank [24] were used for the exposure summary data and constructing the instrumental variables. The summary data for the outcome (fasting GIP) was from the MDC-CC cohort. Out of 147 SNPs in meta-analysis of CARDiOGRAMplusC4D and UK Biobank (ESM Table 1) with $p$ value $<5 \times 10^{-8}$ and $r^{2}$ measure of linkage disequilibrium $<0.2,116$ SNPs were selected with information also in the MDC-CC (ESM Table 2). When running MR analysis, SNPs rs472109 and rs4754698 were removed as their effect alleles were ambiguous. In total, 114 SNPs were utilised to construct the instrumental variables for 2SMR from CAD to fasting GIP. The inverse variance weighted (IVW) method, which is a widely accepted approach for 2SMR analyses with several SNPs as instrumental variables, was used for the main analysis. The sensitivity analyses for the pleiotropy effect was performed using the MR Egger method [25].

\section{Results}

Detailed characteristics of the study populations are presented in Tables 1 and 2.

\section{Total and cardiovascular mortality}

Fasting GIP In Cox regression analyses, adjusted for sex and age (Model 1), each 1 SD increment of log-transformed fasting GIP concentration was associated with higher total mortality risk in both cohorts. To determine the extent to which this was mediated by known risk factors for CVD we further adjusted the analyses for BMI, FPG, fasting insulin, SBP, LDL-cholesterol, HDL-cholesterol and smoking (Model 2 ), and the associations remained significant (Table 3 ). In Model 3, diabetes status, lipid-lowering treatment, BPlowering treatment and educational level were included on top of the covariates in Model 2 (Table 3). The cumulative incidence of total mortality for each quartile of fasting GIP is shown in Kaplan-Meier plots (Fig. 1a, b).

Increased fasting GIP concentration was also associated with risk of cardiovascular mortality in all models (Table 3).

Post-challenge GIP Increased GIP concentrations after a standard OGTT (post-challenge) were associated with higher risk of total and cardiovascular mortality in a meta-analysis of the two cohorts in all models. However, the post-challenge associations were driven mainly by the MDC-CC cohort (Table 3).

Sensitivity analysis To rule out the possibility that the higher mortality risk was a result of individuals being diabetic and hence contributing to a larger extent to total mortality, analyses were carried out on associations of GIP and risk of mortality, as well as cardiovascular mortality, in both cohorts excluding individuals with prevalent diabetes. The associations between GIP and mortality risk essentially remained unchanged (ESM Results, ESM Tables 3-5).

\section{Incident non-fatal cardiovascular events}

Fasting GIP Next, we analysed associations between GIP concentrations and incident, non-fatal CVD. Fasting GIP concentration was associated with incident CVD during a mean follow-up of 8.8 years in all models in PPP-Botnia. In MDC-CC, fasting GIP was not associated with higher risk of incident non-fatal CVD (Table 4).

Post-challenge GIP The post-challenge GIP concentration was associated with non-fatal incident CVD in PPP-Botnia. In MDC-CC, the associations were not significant (Table 4).

A cross-sectional, exploratory analysis of GIP concentrations and CVD prevalence is presented in ESM Results.

Fasting and post-challenge GLP-1 Corresponding analyses were performed for GLP-1 in 3625 subjects but no significant associations were observed for either fasting or post-challenge levels of GLP-1 and mortality risk, or CVD subgroups in the 
MDC-CC study (ESM Table 6). GLP-1 was not measured at the basal visit in the PPP-Botnia cohort.

\section{MR analyses}

We performed a $2 \mathrm{SMR}$ analysis by Wald ratio method between GIP levels as exposure and CAD $(n=184,305 ; 60,801$ cases, 123,504 controls) and myocardial infarction $(n=171,875$; 43,676 cases, 128,199 controls) as outcome variables in CARDIoGRAMplusC4D. The same procedure was applied using data from UK Biobank (CAD: $n=296,525 ; 34,541$ cases, 261,984 controls) [24]. For the exposure (GIP), the initial sample was 3344 individuals of Swedish ancestry; 4905 individuals of Finnish ancestry were used as the replication sample [26]. We utilised rs1800437 as the instrumental variable (see ESM Table 7 for more details). The results show a significant association between fasting GIP and both CAD $(p=0.002)$ and myocardial infarction $(p=0.013)$, as presented in Table 5 using CARDIoGRAMplusC4D data, and significant associations between fasting GIP and CAD using UK Biobank data $(p=0.001)$. Further, a reverse 2 SMR analysis was carried out with CAD as exposure and GIP as outcome variable (Table 5; detailed analysis in ESM Table 8). The nonsignificant 2SMR result using the IVW method ( $p=0.148$ ) shows that there is no directional association from CAD to GIP. There was no evidence of pleiotropy found through the MR Egger method (Table 5; $p=0.595$ ). The single SNP MR estimates using each of the 114 SNPs used in the reverse MR from CAD to GIP can be found in ESM Table 9. The bidirectional MR analysis confirmed the possible direction solely from GIP to CAD.

\section{Discussion}

This observational study demonstrates that high plasma concentration of fasting GIP is associated with higher risk of total and cardiovascular mortality in two general populations. Further, using a 2SMR, we demonstrated an association between increased GIP levels and CAD.

The results from the two studied populations were generally comparable, with the most consistent effect being the association between fasting GIP concentration and mortality risk. The discrepancies found may be explained by the mean age difference between the two populations (72 years for MDC-CC vs 50 years for PPP-Botnia), resulting in fewer outcomes (mortality and cardiovascular mortality) for PPPBotnia and there may be differences in the underlying pathology of CVD at different ages. Other potential reasons are differences in population and lifestyle, and the sources and definitions of CVD in the two studies (self-reported for PPP and register-derived for $\mathrm{MDC}-\mathrm{CC}$ ). However, the associations between increased GIP levels and higher risk of CAD/ myocardial infarction were confirmed using the large CARDIoGRAMplusC4D data in 2SMR analysis.

Recently, Ussher et al. showed that genetic elimination of GIPR improved survival rate and reduced adverse cardiac remodelling following experimental myocardial infarction in mice [10]. Furthermore, epidemiological studies have shown that fasting GIP concentrations are significantly higher in individuals with a history of CVD and GIPR mRNA expression is higher in the arterial wall of individuals with symptoms of CVD [9]. A suggested mediator of the possible cardiovascular detrimental effects of GIP is osteopontin (OPN) [9, 27, 28]. OPN regulates synthesis of extracellular matrix and the proliferation and migration of endothelial and vascular smooth muscle cells during repair and remodelling of blood vessels. OPN also promotes inflammation and recruitment of leucocytes to the vessel wall [29]. Accordingly, plasma OPN has been associated with the presence and severity of CAD in humans [30]. Notably, GIP stimulation increases OPN expression in mouse arteries and individuals with symptomatic CVD have higher plaque expression of GIPR and OPN (also known as SPP1) mRNA. Further, GIP infusion increases plasma concentration of OPN in humans and this effect is strongest in carriers of the minor allele of the GIPR rs10423928 locus [9]. Interestingly, there is also a known CAD locus (rs46522) in the $U B E 2 Z$ gene, suggested to be mediated by a nonsynonymous coding SNP (rs2291725) in the GIP gene, but the effect of this locus on GIP function and expression is still poorly understood $[31,32]$. While recent experimental data do not actually support a direct damaging effect of GIP on cardiac cells [10,33], recent clinical observations in obese individuals with hyperglycaemia and insulin resistance show an association of increased circulating GIP levels with biomarkers of chronic low-grade inflammation (this, in turn, might facilitate CVD) [34].

The MR associations between GIP and CAD shown in our study indicate a direct role for GIPR signalling in the pathways leading to these endpoints, although we cannot conclude that all of the risk increase observed in this study is due to direct effects of GIP on the cardiovascular system. The risk increase for death may, as an example, be mediated by unhealthy fat distribution, independent of insulin levels, that is associated with higher GIP release, or by promotion of obesity [35, 36]. However, our analyses were adjusted for BMI, implicating other pathways. Another possibility is that the association is mediated by effects on glucose homeostasis and risk of diabetes. This was addressed by adjusting for fasting glucose and insulin values in Model 2 and addition of diabetes status in Model 3. We also did a set of analyses wherein we excluded all diabetic individuals (prevalent and incident diabetes cases) in the MDC-CC cohort (ESM Tables 3-5), with associations between GIP and mortality risk essentially unchanged. In the PPP-Botnia cohort, the diabetes status of individuals who did not attend the follow-up visit 
could not be determined. Instead, we analysed risk of incident and total CVD in individuals who were normoglycaemic both at baseline and at follow-up and found that all associations remained in this smaller subset (ESM Table 4). A third possibility is that part of the associations could be due to unmeasured covariates.

The LEADER, SUSTAIN-6, HARMONY and REWIND studies [3-7] found lower rates of cardiovascular events among high-risk individuals with type 2 diabetes treated with the GLP-1 analogues liraglutide, semaglutide, albiglutide and dulaglutide, respectively, vs placebo. In our study, neither fasting nor post-challenge GLP-1 concentrations were associated with the risk of CVD or death, nor did we find any protective effects of GLP-1 on mortality and CVD risk. This discrepancy could be due either to the different populations studied (e.g. the PPP-Botnia and MDC-CC are population cohorts consisting of only $5.9 \%$ and $4.4 \%$ individuals with diabetes, respectively, in contrast to the LEADER and SUSTAIN trial in which only diabetic individuals were studied) or, even more likely, to different concentrations of GLP-1 as the cardioprotective effects of GLP-1 agonists/analogues demonstrated earlier are attributed to pharmacologically induced, supraphysiological levels of GLP-1 in contrast to the normal, physiological GLP-1 levels in our study.

\section{Strength and limitations}

The use of well-characterised, prospective cohorts with many participants and a relatively long follow-up time is a significant strength of the current study. Further, we used nationwide registers with $100 \%$ coverage and high accuracy. We could not completely exclude confounding effects of unmeasured covariates linked to GIP levels but tried to minimise confounders by adjusting for relevant risk factors. Another strength of this study is the demonstration of an effect of a functional genetic variant in GIPR on $\mathrm{CAD}$ /myocardial infarction using the MR approach, suggesting an involvement of the GIP signalling pathway in the pathogenesis of CAD.

We acknowledge that the MR analysis has limitations such as horizontal pleiotropy. To improve the reliability of our GIP to $\mathrm{CAD}$ /myocardial infarction MR analysis, we considered the possible confounding phenotypes and tested for their association with our instrumental variable rs1800437, which is associated with insulin secretion [26, 28, 37], BMI and other related phenotypes (ESM Tables 10 and 11). These phenotypes are likely to mediate at least some of the association between the genetic variant in GIP and CAD (vertical pleiotropy). Because of this, and because the genetic variant affects both the concentration of GIP and the expression and function of its receptor (horizontal pleiotropy), the MR effect size estimates should be interpreted with caution. However, using a genetic variant in GIPR greatly strengthens the evidence that the association is due to GIP signalling, since there are no known alternative ligands for the GIPR. For the reverse MR, there was no evidence of a pleiotropic effect based on MR Egger analyses (Table 5). Furthermore, the two cohorts (PPP-Botnia and MDC-CC) differ regarding the mean age of the participants (those in PPP-Botnia were younger), eventrate and how endpoints were collected, possibly explaining the discrepancies in the results presented. Finally, our data was collected in two Nordic regions, which limits the applicability to other populations.

\section{Conclusion}

In two prospective, community-based studies, elevated levels of GIP were associated with greater risk of all-cause and cardiovascular mortality within 5-9 years of follow-up, whereas GLP-1 levels were not associated with excess risk. Further studies are needed to determine the cardiovascular effects of GIP per se.

Acknowledgements Open access funding provided by Lund University. The Knut and Alice Wallenberg foundation is acknowledged for generous support.

Data availability The data that support the findings of this study are available upon request from the Steering Committee of the Malmö Diet and Cancer study by contacting its chair, O. Melander (olle.melander@med.lu.se). Restrictions apply to the availability of these data, which were used under licence for the current study, and so they are not publicly available due to ethical and legal restrictions related to the Swedish Biobanks in Medical Care Act (2002:297) and the Personal Data Act (1998:204). Data on myocardial infarction have been contributed by CARDIoGRAMplusC4D investigators and have been downloaded from http://www.CARDIOGRAMPLUSC4D.ORG. Data on CAD have been contributed by the CARDIoGRAMplusC4D and UK Biobank CardioMetabolic Consortium CHD working group.

Funding This work was supported by these Funding sources: grants from the Swedish Research Council (K2008 - 65X-20 752-01-3, K2011-65X$20752-04-6,2010-3490$ ), the Lundströms Foundation, the Swedish Heart-Lung Foundation (2010-0244; 2013-0249), ALF government grants (Dnr: 2012/1789), Folkhälsan Research Foundation, Sigrid Jusélius Foundation, the Finnish Medical Society, the Finnish Diabetes research Foundation, the Academy of Finland (FiDiPro grant 263401 and project grant 267882). AJ was funded by Lund University and Region Skåne. MM and OM were supported by grants from the Swedish Medical Research Council, the Swedish Heart and Lung Foundation, the Medical Faculty of Lund University, Skåne University Hospital, the Albert Påhlsson Research Foundation, the Crafoord Foundation, the Ernhold Lundströms Research Foundation, the Region Skåne, the Hulda and Conrad Mossfelt Foundation, the Southwest Skåne Diabetes Foundation, the King Gustaf V and Queen Victoria Foundation, the Lennart Hanssons Memorial Fund, Knut and Alice Wallenberg Foundation and the Marianne and Marcus Wallenberg Foundation. MM was also supported by the Wallenberg Centre for Molecular Medicine at Lund University. PMN was supported by grants from the Swedish Medical Research Council, the Swedish Heart and Lung Foundation, the Medical Faculty of Lund University, Skåne University Hospital and the Ernhold Lundströms Research Foundation. EA was supported by grants from the Swedish Research Council (201702688), ALF government grants, Diabetes Wellness Sweden (25-420 PG), the Swedish Heart and Lung Foundation, the Crafoord Foundation, the 
Swedish Diabetes Foundation, the Novo Nordisk Foundation (NNF18OC0034408), Bo and Kerstin Hjelt Foundation and the Albert Påhlsson Research Foundation. The PPP-Botnia study has been financially supported by grants from the Sigrid Juselius Foundation, Folkhälsan Research Foundation, Ollqvist Foundation, Nordic Center of Excellence in Disease Genetics, EU (EXGENESIS, EUFP7-MOSAIC), Signe and Ane Gyllenberg Foundation, Swedish Cultural Foundation in Finland, Finnish Diabetes Research Foundation, Foundation for Life and Health in Finland, Finnish Medical Society, Paavo Nurmi Foundation, Helsinki University Central Hospital Research Foundation, Perklén Foundation, Närpes Health Care Foundation and Ahokas Foundation. The study has also been supported by the Ministry of Education in Finland, Municipal Health Care Center and Hospital in Jakobstad and healthcare centres in Vasa, Närpes and Korsholm. MFG was supported by the Swedish Heart and Lung Foundation (no. 20160872) and the Swedish Research Council (no. 2014-03352). LMB was supported by The Swedish Society for Medical Research. SR and JD were supported by grants from the Ministry of Economy and Competitiveness, Madrid, Spain (Instituto de Salud Carlos III grants RD12/0042/0009 and PI15/01909) and the European Commission FP7 Programme, Brussels, Belgium (EUMASCARA project grant HEALTH-2011-278249, HOMAGE project grant HEALTH-2012-305507). The funders of the study had no role in study design, data collection, data analysis, data interpretation or writing of the report. AJ, MM, EA and LG had full access to the data in the study and had final responsibility for the decision to submit for publication.

Duality of interest The authors declare that there is no duality of interest associated with this manuscript.

Contribution statement $\mathrm{MM}$ is the guarantor for this manuscript. AJ, NAP, MP, OM, MM, LG, EA, PMN, TT, LH, JJH, SST, LMB, RBP, SR, JD and MFG contributed to the conception of the work and data collection. AJ, NA-P, PWF, MP, OM, PA, MM, LG, EA and PMN contributed to data analysis and interpretation. AJ, NA-P, LG, MM, EA, MFG, LMB, JD, SR, PWF and PMN contributed to drafting the article (MM and EA contributed equally). All authors contributed to the critical revision of the article. All authors gave final approval of the version to be published.

Open Access This article is licensed under a Creative Commons Attribution 4.0 International License, which permits use, sharing, adaptation, distribution and reproduction in any medium or format, as long as you give appropriate credit to the original author(s) and the source, provide a link to the Creative Commons licence, and indicate if changes were made. The images or other third party material in this article are included in the article's Creative Commons licence, unless indicated otherwise in a credit line to the material. If material is not included in the article's Creative Commons licence and your intended use is not permitted by statutory regulation or exceeds the permitted use, you will need to obtain permission directly from the copyright holder. To view a copy of this licence, visit http://creativecommons.org/licenses/by/4.0/

\section{References}

1. Mayo KE, Miller LJ, Bataille D et al (2003) International Union of Pharmacology. XXXV. The glucagon receptor family. Pharmacol Rev 55(1):167-194. https://doi.org/10.1124/pr.55.1.6

2. Pujadas G, Drucker DJ (2016) Vascular biology of glucagon receptor superfamily peptides: mechanistic and clinical relevance. Endocr Rev 37(6):554-583. https://doi.org/10.1210/er.2016-1078

3. Marso SP, Poulter NR, Nissen SE et al (2013) Design of the liraglutide effect and action in diabetes: evaluation of cardiovascular outcome results (LEADER) trial. Am Heart $\mathrm{J}$ 166(5):823-830 e825. https://doi.org/10.1016/j.ahj.2013.07.012

4. Marso SP, Bain SC, Consoli A et al (2016) Semaglutide and cardiovascular outcomes in patients with type 2 diabetes. N Engl J Med 375(19):1834-1844. https://doi.org/10.1056/NEJMoa1607141

5. Marso SP, Daniels GH, Brown-Frandsen K et al (2016) Liraglutide and cardiovascular outcomes in type 2 diabetes. N Engl J Med 375(4):311-322. https://doi.org/10.1056/NEJMoa1603827

6. Hernandez AF, Green JB, Janmohamed S et al (2018) Albiglutide and cardiovascular outcomes in patients with type 2 diabetes and cardiovascular disease (Harmony Outcomes): a double-blind, randomised placebo-controlled trial. Lancet 392(10157):15191529. https://doi.org/10.1016/S0140-6736(18)32261-X

7. Gerstein HC, Colhoun HM, Dagenais GR et al (2019) Dulaglutide and cardiovascular outcomes in type 2 diabetes (REWIND): a double-blind, randomised placebo-controlled trial. Lancet 394(10193):121-130. https://doi.org/10.1016/S0140-6736(19) 31149-3

8. Scott RA, Freitag DF, Li L et al (2016) A genomic approach to therapeutic target validation identifies a glucose-lowering GLPIR variant protective for coronary heart disease. Sci Transl Med 8(341):341ra376. https://doi.org/10.1126/scitranslmed.aad3744

9. Berglund LM, Lyssenko V, Ladenvall C et al (2016) Glucosedependent insulinotropic polypeptide stimulates osteopontin expression in the vasculature via endothelin-1 and CREB. Diabetes 65(1):239-254. https://doi.org/10.2337/db15-0122

10. Ussher JR, Campbell JE, Mulvihill EE et al (2018) Inactivation of the glucose-dependent insulinotropic polypeptide receptor improves outcomes following experimental myocardial infarction. Cell Metab 27(2):450-460 e456. https://doi.org/10.1016/j.cmet. 2017.11.003

11. Nitz I, Fisher E, Weikert $C$ et al (2007) Association analyses of GIP and GIPR polymorphisms with traits of the metabolic syndrome. Mol Nutr Food Res 51(8):1046-1052. https://doi.org/10.1002/ mnfr.200700048

12. Isomaa B, Forsen B, Lahti K et al (2010) A family history of diabetes is associated with reduced physical fitness in the Prevalence, Prediction and Prevention of Diabetes (PPP)-Botnia study. Diabetologia 53(8):1709-1713. https://doi.org/10.1007/s00125010-1776-y

13. Rosvall M, Persson M, Ostling G et al (2015) Risk factors for the progression of carotid intima-media thickness over a 16-year follow-up period: the Malmo Diet and Cancer Study. Atherosclerosis 239(2):615-621. https://doi.org/10.1016/j. atherosclerosis.2015.01.030

14. Alberti KG, Zimmet PZ (1998) Definition, diagnosis and classification of diabetes mellitus and its complications. Part 1: diagnosis and classification of diabetes mellitus provisional report of a WHO consultation. Diabet Med 15(7):539-553. https://doi.org/10.1002/ (SICI)1096-9136(199807)15:7<539::AID-DIA668>3.0.CO;2-S

15. Jujic A, Nilsson PM, Persson M et al (2016) Atrial natriuretic peptide in the high normal range is associated with lower prevalence of insulin resistance. J Clin Endocrinol Metab 101(4):13721380. https://doi.org/10.1210/jc.2015-3518

16. (Socialstyrelsen) TNBoHaW (2000) Evaluation of quality of diagnosis of acute myocardial infarction, inpatient register 1997 and 1995. Socialstyrelsen, Stockholm [In Swedish]

17. Engstrom G, Jerntorp I, Pessah-Rasmussen H, Hedblad B, Berglund G, Janzon L (2001) Geographic distribution of stroke incidence within an urban population: relations to socioeconomic circumstances and prevalence of cardiovascular risk factors. Stroke 32(5):1098-1103

18. Hammar N, Alfredsson L, Rosen M, Spetz CL, Kahan T, Ysberg AS (2001) A national record linkage to study acute myocardial infarction incidence and case fatality in Sweden. Int J Epidemiol 30(Suppl 1):S30-S34 
19. Ingelsson E, Arnlov J, Sundstrom J, Lind L (2005) The validity of a diagnosis of heart failure in a hospital discharge register. Eur J Heart Fail 7(5):787-791. https://doi.org/10.1016/j.ejheart.2004.12.007

20. R Core Team (2013) R: a language and environment for statistical computing. R Foundation for Statistical Computing Vienna. Available from: http://www.R-project.org/ (accessed 20 May 2019)

21. Yavorska OO, Burgess S (2017) MendelianRandomization: an R package for performing Mendelian randomization analyses using summarized data. Int J Epidemiol 46(6):1734-1739. https://doi.org/ 10.1093/ije/dyx034

22. Hemani G, Zheng J, Elsworth B, et al. (2018) The MR-Base platform supports systematic causal inference across the human phenome. Elife 7. https://doi.org/10.7554/eLife.34408

23. Viechtbauer W (2010) Conducting meta-analyses in $\mathrm{R}$ with the metafor package. J Stat Software 36(3):48. https://doi.org/10. 18637 /jss.v036.i03

24. van der Harst P, Verweij N (2018) Identification of 64 novel genetic loci provides an expanded view on the genetic architecture of coronary artery disease. Circ Res 122(3):433-443. https://doi.org/10. 1161/CIRCRESAHA.117.312086

25. Burgess S, Bowden J, Fall T, Ingelsson E, Thompson SG (2017) Sensitivity analyses for robust causal inference from mendelian randomization analyses with multiple genetic variants. Epidemiology 28(1):30-42. https://doi.org/10.1097/EDE. 0000000000000559

26. Almgren P, Lindqvist A, Krus U, et al. (2017) Genetic determinants of circulating GIP and GLP-1 concentrations. JCI Insight 2(21): 93306. https://doi.org/10.1172/jci.insight.93306

27. Ahlqvist E, Osmark P, Kuulasmaa T et al (2013) Link between GIP and osteopontin in adipose tissue and insulin resistance. Diabetes 62(6):2088-2094. https://doi.org/10.2337/db12-0976

28. Lyssenko V, Eliasson L, Kotova O et al (2011) Pleiotropic effects of GIP on islet function involve osteopontin. Diabetes 60(9):2424 2433. https://doi.org/10.2337/db10-1532

29. Wolak T (2014) Osteopontin - a multi-modal marker and mediator in atherosclerotic vascular disease. Atherosclerosis 236(2):327337. https://doi.org/10.1016/j.atherosclerosis.2014.07.004
30. Ohmori R, Momiyama Y, Taniguchi H et al (2003) Plasma osteopontin levels are associated with the presence and extent of coronary artery disease. Atherosclerosis 170(2):333-337. https://doi. org/10.1016/s0021-9150(03)00298-3

31. Schunkert H, Konig IR, Kathiresan S et al (2011) Large-scale association analysis identifies 13 new susceptibility loci for coronary artery disease. Nat Genet 43(4):333-338. https://doi.org/10.1038/ ng. 784

32. Chang CL, Cai JJ, Lo C, Amigo J, Park JI, Hsu SY (2011) Adaptive selection of an incretin gene in Eurasian populations. Genome Res 21(1):21-32. https://doi.org/10.1101/gr.110593.110

33. Hiromura M, Mori Y, Kohashi K et al (2016) Suppressive effects of glucose-dependent insulinotropic polypeptide on cardiac hypertrophy and fibrosis in angiotensin II-infused mouse models. Circ J 80(9):1988-1997. https://doi.org/10.1253/circj.CJ-16-0152

34. Goralska J, Razny U, Polus A et al (2018) Pro-inflammatory gene expression profile in obese adults with high plasma GIP levels. Int $\mathrm{J}$ Obes 42(4):826-834. https://doi.org/10.1038/ijo.2017.305

35. Moller CL, Vistisen D, Faerch K et al (2016) Glucose-dependent insulinotropic polypeptide is associated with lower low-density lipoprotein but unhealthy fat distribution, independent of insulin: the ADDITION-PRO study. J Clin Endocrinol Metab 101(2):485493. https://doi.org/10.1210/jc.2015-3133

36. Morgan LM, Hampton SM, Tredger JA, Cramb R, Marks V (1988) Modifications of gastric inhibitory polypeptide (GIP) secretion in man by a high-fat diet. Br J Nutr 59(3):373-380. https://doi.org/10. 1079/bjn19880046

37. Saxena R, Hivert MF, Langenberg C et al (2010) Genetic variation in GIPR influences the glucose and insulin responses to an oral glucose challenge. Nat Genet 42(2):142-148. https://doi.org/10. 1038/ng.521

Publisher's note Springer Nature remains neutral with regard to jurisdictional claims in published maps and institutional affiliations. 